\title{
ODONTOLOGIA E DOENÇA DE ALZHEIMER: UMA REVISÃO INTEGRATIVA
}

\author{
ODONTOLOGIA E DOENÇA DE ALZHEIMER: UMA REVISÃO \\ INTEGRATIVA
}

\author{
Clara Bhertyne Pinheiro Romão ${ }^{1}$ \\ Rafaela Costa de Holanda ${ }^{2}$ \\ Samara Albuquerque de Souza ${ }^{3}$ \\ Layana Oliveira de Abreu ${ }^{4}$ \\ Marcos Alexandre Casimiro de Oliveira ${ }^{5}$ \\ Clarissa Lopes Drumond6
}

RESUMO: Introdução: A doença de Alzheimer (DA) é caracterizada por uma afecção neurodegenerativa progressiva e irreversível, que a priori gera perda da memória recente e posteriormente, a memória geral, incluindo vários distúrbios cognitivos, o que afeta diretamente a convivência do indivíduo. Para facilitar sua compreensão diagnóstica e o tratamento, é dividida em três estágios: inicial, intermediário e avançado. Objetivo: Nesse contexto, o objetivo deste trabalho foi realizar uma revisão integrativa de literatura sobre a odontologia e a doença de Alzheimer com ênfase no manejo e tratamento odontológico em consultório. Método: Para isso, o método utilizado foi o qualitativo, sendo realizada uma revisão integrativa de literatura a partir de pesquisas bibliográficas nas bases de dados: SCiELO (Scientific Online Library), Google Acadêmico, PUBMED (National Library of Medicine National Institutes of Health dos EUA) e o Guia de atenção à saúde bucal da pessoa com deficiência (Ministério da Saúde), a partir de trabalhos publicados entre 2006 e 2020. Resultados e discussão: A partir desse levantamento, fica evidente a importância de o cirurgião-dentista ter um conhecimento voltado para a DA, sendo necessário a aplicação de técnicas e manejos adequados para a condução do tratamento odontológico, tornando o atendimento satisfatório e garantindo a saúde bucal e a qualidade de vida do paciente. Conclusão: Portanto, embora haja estudos e protocolos voltados ao tratamento odontológico e a doença

\footnotetext{
${ }^{1}$ Graduanda em Odontologia da Faculdade Santa Maria - FSM.

2 Mestra em Odontologia. Professora da Faculdade Santa Maria (FSM).

${ }^{3}$ Graduanda em Odontologia da Faculdade Santa Maria - FSM.

${ }^{4}$ Graduanda em Odontologia da Faculdade Santa Maria - FSM.

${ }^{5}$ Mestre em Ciências Odontológicas. Docente da Faculdade Santa Maria (FSM).

${ }^{6}$ Doutora em Odontologia. Professora e Coordenadora do curso de Odontologia da Faculdade Santa Maria (FSM).
} 
de Alzheimer (DA), se faz necessário um protocolo mais robusto e objetivo que oriente o profissional de odontologia para o manejo adequado do paciente com a DA, permitindo assim uma maior uniformidade e qualidade nesse atendimento.

Palavras chave: saúde bucal, doença de Alzheimer, assistência odontológica.

ABSTRACT: Introduction: Alzheimer's disease $(A D)$ is characterized by a progressive and irreversible neurodegenerative disorder, which a priori generates loss of 1081erviss memory and later, general memory, including several cognitive disorders, which directly affects the individual's life. To facilitate its diagnostic understanding and treatment, it is divided into three stages: initial, intermediate and advanced. Objective: In this 1081ervisse, the aim of this study was to carry out na integrative literature review on dentistry and Alzheimer's disease with emphasis on dental management and treatment in the office. Method: For this, the method used was qualitative, and na integrative literature review was carried out based on bibliographic research in the following databases: SCIELO (Scientific Online Library), Academic Google, PUBMED (National Library of Medicine, National Institutes of Health, USA) and the Guide for oral health care for people with disabilities (Ministry of Health), based on studies published between 2006 and 2020. Results and discussion: From this survey, it is 1081ervisse the importance of the dental surgeon having knowledge focused on $A D$, requiring the application of appropriate techniques and management to conduct dental treatment, making the 1081ervisse satisfactory and ensuring oral health and quality of the patient's life. Conclusion: Therefore, although there are studies and protocols aimed at dental treatment and Alzheimer's disease (AD), a more robust and objective protocol is needed to guide dental professionals in the proper management of patients with $A D$, thus allowing for greater uniformity and quality in this 1081ervisse.

Keywords: oral health, Alzheimer's disease, dental care. 


\section{INTRODUÇÃO}

Atualmente, a doença do Alzheimer (DA) é um dos maiores desafios a serem enfrentados no campo da saúde pública, visto que os sinais e sintomas se apresentam de forma gradual e de rápida progressão nos indivíduos diagnosticados. $\mathrm{Na}$ progressão da doença, essas pessoas ficam sujeitas ao cuidado de terceiros, ou seja, perdem a capacidade de independência motora e cognitiva (SERENIKII; BARBATO FRAZÃO VITAL, 2017).

O Alzheimer foi caracterizado pelo neuropatologista alemão Alois Alzheimer no ano de 1907, como uma afecção neurodegenerativa progressiva e irreversível, que gera inicialmente a perda de memória recente, mas o indivíduo pode se lembrar com precisão dos fatos antigos, onde afeta de maneira direta a capacidade de aprendizado, compreensão, orientação, atenção e linguagem (BATISTA, 2018).

A DA é caracteriza por uma alteração neurodegenerativa progressiva, fatal e até hoje, sem procedimentos de cura. Promove a perda da função intelectual, habilidades de percepção e, geralmente, surge após os 60 anos do indivíduo, podendo aparecer os primeiros e quase imperceptíveis sintomas antes da idade programada. Esses pacientes, tendem a perder a capacidade de se preocuparem consigo e, em estágios mais avançados, perdem a mobilidade total e como consequência, chegam ao óbito (FRIEDLANDER et al., 2006).

O Alzheimer pode ser diagnosticado por sinais clínicos e por sintomas primários, como, a percepção do tempo e do lugar, testes de memória, avaliação da linguagem e da escrita. Vale ressaltar também, que deve conter o diagnóstico diferencial, através do descarte de outras doenças como, depressão, aumento da ingestão de medicamentos, acidentes vasculares encefálicos, entre outras (FRIEDLANDER et al., 2006).

Para facilitar a compreensão da DA, ela é dividida em três fases: leve, moderada e grave. Essa divisão irá contribuir para identificar em qual fase o paciente 
se encontra e como o cirurgião-dentista deve atuar diante dos atendimentos (OLIVEIRA; D’ÁVILA, 2019).

Sabemos que a saúde bucal é inseparável da saúde geral, ou seja, para o funcionamento ideal do corpo requer as duas combinadas para garantir o bem-estar ao indivíduo. Warmling et al (2016), afirmam que devido ao avanço da enfermidade, as pessoas com a DA tendem a ter uma condição bucal desfavorável, por muitas vezes, não conseguirem realizar sua higienização bucal e podem entrar no grupo de risco das doenças bucais e da perda precoce dos dentes pela ausência da escovação ou profilaxia adequada. Desse modo, os pacientes com Alzheimer necessitam de um cuidador ativo e extremamente atencioso, pois este deve ter instruções claras de higiene, educação em saúde e noções de execuções dos procedimentos orais básicos.

A abordagem em pacientes com a DA, vai além da anamnese, inicia pelo exame físico extrabucal, onde vai avaliar a presença de linfonodos, tônus muscular, lesões de face, os lábios e as comissuras. Já na parte intrabucal, verifica-se a mucosa, saliva, e dentes, o que incluem a saúde bucal do indivíduo. Nos idosos desdentados totais e parciais, onde fazem uso das próteses dentárias, necessitamos observar condições de higiene, fraturas na peça protética, desgastes, adaptação e funcionalidade (SPEZZIA, 2015).

Os principais problemas bucais encontrados em pacientes com Alzheimer são, candidíase nas suas diversas formas clínicas, língua saburrosa, lesões por próteses mal adaptadas, como, úlceras e hiperplasia inflamatória, cárie radicular e doença periodontal. Devido ao acúmulo de biofilme, observamos uma crescente deficiência na escovação e higienização das peças protéticas, o que induz aos altos índices de achados de doenças nos pacientes com Alzheimer. Observa-se também, casos de hipossalivação, onde por muitas vezes são reflexos da medicação ingerida pelo idoso. É importante salientar, que a progressão das doenças bucais, geralmente ocorre pela perda do autocuidado. Portanto, quanto antes for feito o diagnóstico demencial, mais rápido será o tratamento e acabará evitando complicações na saúde bucal (SPEZZIA, 2015).

Geralmente, o primeiro contato do paciente com Alzheimer e do cirurgiãodentista, acontece de forma tardia, até porque, a doença é caracterizada pela idade 
avançada e, os pacientes acabam não fazendo acompanhamentos contínuos com o mesmo dentista, o que resulta na dificuldade do atendimento. Por isso, é primordial uma anamnese detalhada contendo todas as informações do paciente, onde em casos mais severos, precisamos das respostas do cuidador ou familiar que convive com indivíduo (OLIVEIRA; D'ÁVILA, 2019).

O cirurgião-dentista frequentemente irá se encontrar em duas situações nos atendimentos aos pacientes com Alzheimer (VARJÃO, 2006). A primeira é quando o paciente já tinha à doença com um bom estado de saúde bucal e estava habituado a visitar ao consultório odontológico. Nessas situações, o hábito de higiene deve ser mantido e feito por supervisão do cuidador, para preservação do estado de saúde bucal. Já na segunda situação, é quando já existia uma condição adversa antes da doença, ou seja, o paciente não tinhas bons hábitos de higiene. Nesses casos, o prognóstico será complexo e necessitará de mais auxílio de terceiros, pois além da maior dificuldade em melhorar a condição, não existe a familiaridade do paciente com o tratamento e, dependendo do estágio da DA, só é possível a realização de tratamentos emergenciais.

De acordo com o contexto apresentado, faz-se necessário que os cuidadores e familiares redobrem a atenção a saúde bucal dos idosos que sofrem de Alzheimer e que sejam assistidos por profissionais especializados e capacitados na área, que são os cirurgiões-dentistas (BARBOSA et al., 2021).

A temática discutida, nesse estudo, é de extrema relevância para a melhoria da qualidade dos atendimentos aos pacientes que sofrem de Alzheimer e para os cuidadores que enfrentam dificuldades em executar suas funções. Acrescido a isso, devemos destacar, a importância para os atuais e futuros profissionais de odontologia, a fim de buscar manejos adequados e humanizados para o atendimento desses pacientes. Dessa forma, justifica-se a necessidade de estudos sobre as principais doenças bucais que afetam os portadores da DA, para facilitar o diagnóstico, conduta adequada e promover um atendimento individualizado e humanizado aos pacientes.

Logo, este estudo é necessário para buscar evidências cientificas sobre prevenção das doenças bucais como, cáries, gengivite, periodontite, candidíase entre outras, higienização e condutas adequadas nos pacientes com DA geradas 
pelo cirurgião-dentista. Deste modo, o objetivo desse trabalho é apresentar as características da DA e os principais problemas bucais encontrados nesses pacientes, no sentido de auxiliar na abordagem clínica do cirurgião-dentista no tratamento odontológico.

\section{METODOLOGIA}

Trata-se de uma revisão integrativa de literatura que tem como finalidade realizar uma revisão integrativa de literatura sobre a odontologia e a doença de Alzheimer com ênfase no manejo e tratamento odontológico em consultório.

Para obter os resultados satisfatórios sobre o assunto em questão, foi realizada uma revisão bibliográfica, entre os meses de junho a outubro de 2021, nas bases de dados LILACs (Literatura Latino-Americana e do Caribe em Ciências da Saúde), SCiELO (Scientific Online Library), Google Acadêmico e PUBMED (National Library of Medicine National Institutes of Health dos EUA).

As palavras-chave utilizadas em português foram "saúde oral", "doença de Alzheimer", "assistência odontológica", "lesões bucais", e no idioma inglês foram: "oral health", "alzheimer disease", "oral lesions" e "delivery of health care", separadas ou associadas, para fazer as chaves de busca pelos operadores booleanos AND e OR.

$\mathrm{Na}$ análise, foram incluídos artigos publicados a partir de 2006 até 2021, resumos disponíveis nos idiomas português e inglês e os que se apresentarem dentro da temática em questão. Foram excluídos artigos em que o resumo não correspondeu com os objetivos do estudo, artigos duplicados nas bases de dados, não disponíveis de forma integral, artigos baseados em relatórios de opinião profissional/relatos de experiência, teses e dissertações. 


\section{RESULTADOS E DISCUSSÃO}

Para a obtenção dos resultados desta pesquisa, foram inseridos artigos publicados entre os anos de 2006 e 2021, sendo que a priori, foram selecionados 49 artigos, sendo 11 artigos incluídos (Quadro 01) na revisão de literatura por atender aos critérios e manter a relevância no estudo (Figura 01).

Figura 01 - Fluxograma das etapas de seleção dos artigos a serem discutidos nesta revisão integrativa.

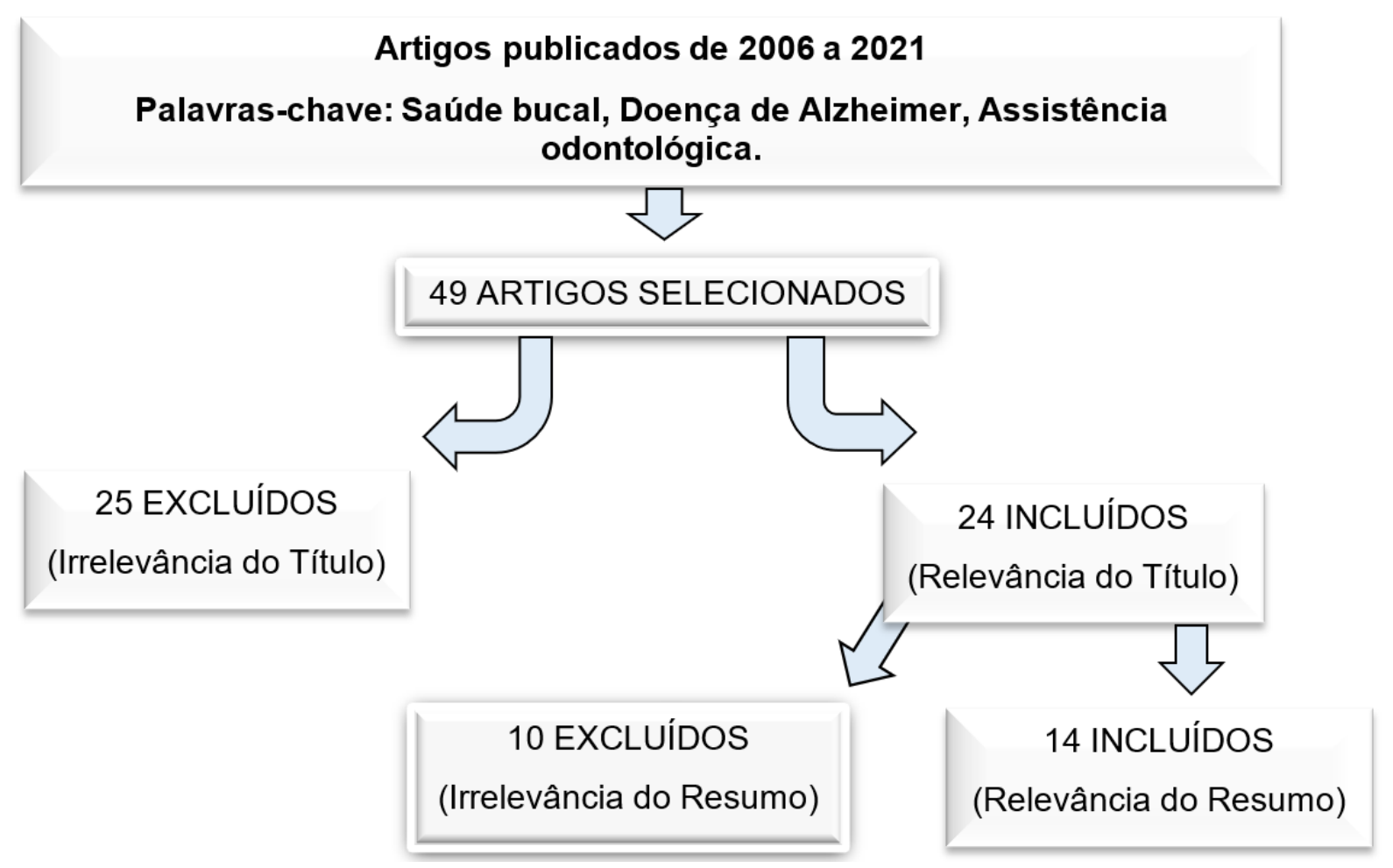

Fonte: Elaborado pelo autor (2021). 
Quadro 1. Artigos selecionados para revisão de literatura no período de 2006 2021.

\begin{tabular}{|c|c|c|c|c|}
\hline $\begin{array}{l}\text { AUTOR- } \\
\text { ANO }\end{array}$ & $\begin{array}{l}\text { TIPO DE } \\
\text { ESTUDO }\end{array}$ & TÍTULO & OBJETIVO & CONCLUSÃO \\
\hline $\begin{array}{l}\text { GALLISA, } \\
2006 .\end{array}$ & $\begin{array}{l}\text { Revisão de } \\
\text { literatura. }\end{array}$ & $\begin{array}{l}\text { Alzheimer na } \\
\text { clínica } \\
\text { odontológica. }\end{array}$ & $\begin{array}{lr}\text { Relatar } & \text { as } \\
\text { repercussões bucais } \\
\text { causadas pela DA e } \\
\text { como O CD deve } \\
\text { conduzir } \quad \text { os } \\
\text { atendimentos diante } \\
\text { desses idosos. }\end{array}$ & $\begin{array}{l}\text { O CD tem a função de preservar } \\
\text { e restaurar a saúde bucal dos } \\
\text { pacientes com DA e retardar a } \\
\text { progressão das doenças } \\
\text { oportunistas nesses pacientes, } \\
\text { estabelecendo protocolos } \\
\text { confiáveis e seguros. }\end{array}$ \\
\hline $\begin{array}{l}\text { VARJÃO, } \\
2006 .\end{array}$ & $\begin{array}{l}\text { Revisão de } \\
\text { literatura. }\end{array}$ & $\begin{array}{l}\text { Assistência } \\
\text { odontológica } \\
\text { para } \\
\text { pacientes } \\
\text { portadores } \\
\text { da DA. }\end{array}$ & $\begin{array}{lr}\text { Apresentar } & \\
\text { informações aos } \\
\text { profissionais de } \\
\text { odontologia a } \\
\text { respeito das técnicas } \\
\text { e procedimentos a } \\
\text { serem realizados nos } \\
\text { pacientes com } \\
\text { Alzheimer. } \\
\end{array}$ & $\begin{array}{l}\text { Exibir aos familiares que quanto } \\
\text { mais eles conhecem a doença, } \\
\text { melhor é a evolução do quadro } \\
\text { dos idosos e mostrar aos CD } \\
\text { que o papel mais importante é } \\
\text { preservar a função oral e } \\
\text { minimizar a progressão de } \\
\text { possíveis doenças. }\end{array}$ \\
\hline $\begin{array}{l}\text { SOUZA et } \\
\text { al., } 2006 .\end{array}$ & $\begin{array}{l}\text { Análise } \\
\text { temática }\end{array}$ & $\begin{array}{l}\text { Doença de } \\
\text { Alzheimer: } \\
\text { protocolo de } \\
\text { atendimento } \\
\text { odontológico. }\end{array}$ & $\begin{array}{l}\text { Revisar os aspectos } \\
\text { importantes r da } \\
\text { saúde bucal dos } \\
\text { portadores de } \\
\text { Alzheimer e o papel } \\
\text { do CD no cuidado } \\
\text { desses pacientes. } \\
\end{array}$ & $\begin{array}{l}\text { A doença de Alzheimer deve ser } \\
\text { compreendida pelos CD para } \\
\text { ser direcionado um tratamento } \\
\text { adequado aos pacientes, pois } \\
\text { eles têm mais predisposição a } \\
\text { terem doenças orais pela } \\
\text { ausência da higienização bucal. }\end{array}$ \\
\hline $\begin{array}{l}\text { FRIEDLA } \\
\text { ND et al., } \\
2008 .\end{array}$ & $\begin{array}{l}\text { Artigo de } \\
\text { revisão }\end{array}$ & $\begin{array}{l}\text { A doença de } \\
\text { Alzheimer: } \\
\text { aspectos } \\
\text { fisiopatológic } \\
\text { os e } \\
\text { farmacológic } \\
\text { os. }\end{array}$ & 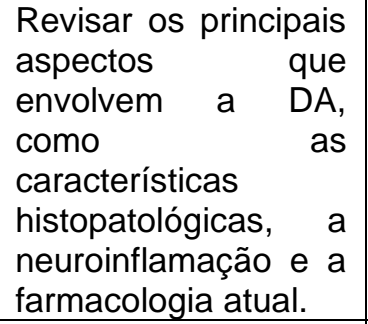 & $\begin{array}{l}\text { Os resultados sugerem que a } \\
\text { neuroinflamação possa exercer } \\
\text { um papel inicial na patogênese } \\
\text { da DA, mas outros estudos } \\
\text { estão sendo concluídos e novas } \\
\text { drogas estão sendo testadas } \\
\text { para garantir a eficácia do } \\
\text { tratamento. }\end{array}$ \\
\hline $\begin{array}{l}\text { SANTIAG } \\
\text { O et al., } \\
2008 .\end{array}$ & $\begin{array}{l}\text { Revisão de } \\
\text { literatura. }\end{array}$ & $\begin{array}{l}\text { A saúde oral } \\
\text { na doença } \\
\text { de } \\
\text { Alzheimer. }\end{array}$ & $\begin{array}{lr}\text { Elaboração de } & \text { um } \\
\text { plano de tratamento } \\
\text { eficaz para } & \text { os } \\
\text { portadores } & \text { de } \\
\text { Alzheimer, visto que } \\
\text { os dentistas devem } \\
\text { educar } & \text { e } \\
\text { supervisionar } & \text { o } \\
\text { paciente } & \text { e } \\
\text { cuidador, } & \text { para } \\
\text { manter a qualidade } \\
\text { de vida r dos } \\
\text { pacientes. } & \\
\end{array}$ & $\begin{array}{l}\text { É imprescindível manter os } \\
\text { estudos sobre o Alzheimer para } \\
\text { garantir os tratamentos mais } \\
\text { avançados a esses pacientes, } \\
\text { sejam preventivos r ou } \\
\text { interventivos, para impedir } \\
\text { complicações fráras, e promover } \\
\text { desnecessárias, e duturas } \\
\text { uma qualidade de vida } \\
\text { adequada e digna. }\end{array}$ \\
\hline $\begin{array}{l}\text { MIRAND } \\
\text { A et al., } \\
2010 .\end{array}$ & $\begin{array}{l}\text { Revisão de } \\
\text { literatura }\end{array}$ & $\begin{array}{l}\text { Doença de } \\
\text { Alzheimer: } \\
\text { característica } \\
\text { s e } \\
\text { orientações } \\
\text { em }\end{array}$ & $\begin{array}{lr}\text { Abordar } & \text { as } \\
\text { características } & \text { e } \\
\text { sugerir } & \text { orientações } \\
\text { no } & \text { atendimento } \\
\text { odontológico } & \text { nas } \\
\text { fases de evolução da }\end{array}$ & $\begin{array}{l}\text { O CD teve ter conhecimento dos } \\
\text { estágios para um tratamento } \\
\text { correto, baseado em qualidade } \\
\text { e prevenção. Os profissionais } \\
\text { em odontologia devem estar } \\
\text { inclusos nas equipes }\end{array}$ \\
\hline
\end{tabular}




\begin{tabular}{|c|c|c|c|c|}
\hline & & odontologia. & doença. & $\begin{array}{l}\text { multiprofissionais. Além disso, } \\
\text { os dentistas devem manter uma } \\
\text { relação com os familiares e } \\
\text { cuidadores para estimulá-los a } \\
\text { aprender sobre a saúde bucal. }\end{array}$ \\
\hline $\begin{array}{l}\text { DIAS et } \\
\text { al., } 2011 .\end{array}$ & $\begin{array}{l}\text { Relato de } \\
\text { caso. }\end{array}$ & $\begin{array}{l}\text { Atendimento } \\
\text { de pacientes } \\
\text { com doença } \\
\text { de Alzheimer } \\
\text { na clínica } \\
\text { odontológica: } \\
\text { desafios e } \\
\text { diretrizes. }\end{array}$ & $\begin{array}{lr}\text { Identificar } & \\
\text { possibilidades } & \text { da } \\
\text { cooperação } & \text { no } \\
\text { tratamento } & \text { dos } \\
\text { idosos com DA, bem } \\
\text { como } & \text { o } \\
\text { desenvolvimento } & \\
\text { familiar, visando os } \\
\text { melhores resultados. }\end{array}$ & $\begin{array}{l}\text { Para o tratamento para o } \\
\text { Alzheimer ter eficácia, os } \\
\text { familiares e cuidadores devem } \\
\text { estar envolvidos cooperando } \\
\text { para atingir o sucesso } \\
\text { terapêutico. }\end{array}$ \\
\hline $\begin{array}{l}\text { ABRITTA } \\
\text { et al., } \\
2012 .\end{array}$ & $\begin{array}{l}\text { Relato de } \\
\text { caso. }\end{array}$ & $\begin{array}{l}\text { Atendimento } \\
\text { multidisciplin } \\
\text { ar em } \\
\text { paciente } \\
\text { idosa com } \\
\text { Alzheimer } \\
\text { em fase } \\
\text { intermediária } \\
\text { em domicílio. }\end{array}$ & $\begin{array}{lr}\text { Apresentar um } & \text { caso } \\
\text { clínico de uma } \\
\text { paciente } & \text { com } \\
\text { osteoporose r } & \text { e } \\
\text { Alzheimer na } & \text { fase } \\
\text { intermediária, } & \text { que } \\
\text { recebe assistência } \\
\text { em saúde em âmbito } \\
\text { domiciliar com a } \\
\text { participação } \\
\text { odontólogo do } \\
\text { equipe } \\
\text { multiprofissional. }\end{array}$ & $\begin{array}{l}\text { O CD deve estar inserido na } \\
\text { equipe multiprofissional tanto no } \\
\text { ambulatorial quanto no } \\
\text { domiciliar na assistência a } \\
\text { pacientes com DA, pois irão } \\
\text { proporcionar um atendimento } \\
\text { digno de saúde com a } \\
\text { promoção de bem-estar e } \\
\text { qualidade de vida. }\end{array}$ \\
\hline $\begin{array}{l}\text { BURLÁ et } \\
\text { al., } 2013 .\end{array}$ & $\begin{array}{l}\text { Revisão de } \\
\text { Literatura. }\end{array}$ & $\begin{array}{l}\text { Panorama } \\
\text { prospectivo } \\
\text { das } \\
\text { demências } \\
\text { no Brasil: um } \\
\text { enfoque } \\
\text { demográfico. }\end{array}$ & $\begin{array}{l}\text { Analisar em } \\
\text { pacientes autistas as } \\
\text { abordagens } \\
\text { educacionais } \\
\text { comportamentais } \\
\text { para tornar o } \\
\text { atendimento } \\
\text { odontológico bem- } \\
\text { sucedido. }\end{array}$ & $\begin{array}{l}\text { Dedicar um tempo para } \\
\text { entender as crianças com TEA, } \\
\text { torna o atendimento } \\
\text { odontológico de alta qualidade. }\end{array}$ \\
\hline $\begin{array}{l}\text { SPEZZIA, } \\
2015 .\end{array}$ & $\begin{array}{l}\text { Revisão de } \\
\text { literatura }\end{array}$ & $\begin{array}{l}\text { Demência e } \\
\text { saúde bucal. }\end{array}$ & $\begin{array}{lr}\text { Realizar } & \text { uma } \\
\text { atualização } & \text { dos } \\
\text { estudos sobre as } \\
\text { repercussões bucais } \\
\text { causadas pelas } \\
\text { demências. }\end{array}$ & $\begin{array}{l}\text { Planejamento } \\
\text { adequado com ênfológico } \\
\text { prevenção e no diagnóstico } \\
\text { correto, pode refletir na melhora } \\
\text { da qualidade de vida desses } \\
\text { pacientes. }\end{array}$ \\
\hline $\begin{array}{l}\text { WARMLI } \\
\text { NG et al., } \\
2016 .\end{array}$ & $\begin{array}{l}\text { Estudo } \\
\text { transversal }\end{array}$ & $\begin{array}{l}\text { Estratégias } \\
\text { de cuidado } \\
\text { bucal para } \\
\text { idosos com } \\
\text { doença de } \\
\text { Alzheimer no } \\
\text { domicílio. }\end{array}$ & $\begin{array}{l}\text { Identificar estratégias } \\
\text { utilizadas no cuidado } \\
\text { à saúde bucal de } \\
\text { idosos com a doença } \\
\text { de Alzheimer no } \\
\text { domicílio. }\end{array}$ & $\begin{array}{l}\text { As estratégias identificadas } \\
\text { serão usadas na criação de } \\
\text { protocolos específicos para } \\
\text { cada paciente, e para facilitar os } \\
\text { cuidados dos familiares. }\end{array}$ \\
\hline $\begin{array}{l}\text { BATISTA, } \\
2018 .\end{array}$ & $\begin{array}{l}\text { Revisão de } \\
\text { literatura. }\end{array}$ & $\begin{array}{l}\text { O uso de } \\
\text { novas } \\
\text { tecnologias } \\
\text { no controle } \\
\text { da doença } \\
\text { de } \\
\text { Alzheimer. }\end{array}$ & $\begin{array}{lr}\text { Destacar } & a \\
\text { importância r da } \\
\text { aplicação de novas } \\
\text { tecnologias romo } \\
\text { ferramentas para } \\
\text { reduzir os impactos } \\
\text { da DA em idosos. }\end{array}$ & $\begin{array}{l}\text { As ferramentas tecnológicas } \\
\text { amenizam os estágios do } \\
\text { Alzheimer e o idoso deve fazer } \\
\text { um acompanhamento social e } \\
\text { institucional junto com a família } \\
\text { e o cuidador. }\end{array}$ \\
\hline $\begin{array}{l}\text { OLIVEIR } \\
A ;\end{array}$ & $\begin{array}{l}\text { Cartilha do } \\
\text { Ministério }\end{array}$ & $\begin{array}{lr}\text { Guia } & \mathrm{de} \\
\text { atencão } & \text { à }\end{array}$ & $\begin{array}{l}\text { Oferecer } \\
\text { informacões aos }\end{array}$ & $\begin{array}{l}\text { O cuidado odontológico das } \\
\text { pessoas com deficiência precisa }\end{array}$ \\
\hline
\end{tabular}




\begin{tabular}{|c|c|c|c|c|}
\hline $\begin{array}{l}\text { D'ÁVILA, } \\
2019 .\end{array}$ & da saúde & $\begin{array}{l}\text { saúde bucal } \\
\text { da pessoa } \\
\text { com } \\
\text { deficiência. }\end{array}$ & $\begin{array}{lr}\text { profssionais da rede } \\
\text { de atenção } & \text { SUS, } \\
\text { promovendo } & \text { a } \\
\text { qaulificação } & \text { dos } \\
\text { atendimentos } & \\
\text { odontológicos a } \\
\text { partir de estratégias } \\
\text { para manejo e o } \\
\text { cuidado, de forma } \\
\text { qualificada e segura. }\end{array}$ & $\begin{array}{l}\text { ser planejado juntamente com } \\
\text { outras intervenções } \\
\text { terapêuticas, priorizando a } \\
\text { promoção da saúde e a } \\
\text { prevenção de agravos e nortear } \\
\text { o trabalho das equipes } \\
\text { multiprofissionais em todas as } \\
\text { etapas do cuidado e do do } \\
\text { processo de reabilitação e nos } \\
\text { diferentes pontos da Rede de } \\
\text { Atencão à Saúde do SUS. }\end{array}$ \\
\hline $\begin{array}{l}\text { BARBOS } \\
\text { A et al., } \\
2021 .\end{array}$ & $\begin{array}{l}\text { Revisão } \\
\text { sistemática. }\end{array}$ & $\begin{array}{l}\text { Práticas de } \\
\text { saúde oral } \\
\text { em idosos } \\
\text { com } \\
\text { demência: } \\
\text { Revisão } \\
\text { sistemática }\end{array}$ & \begin{tabular}{lr} 
Como & são \\
conduzidas & as \\
praticas & \\
odontologicas & em \\
pacientes idosos \\
\multicolumn{2}{l}{ com demencia }
\end{tabular} & $\begin{array}{l}\text { Os resultados obtidos relatam a } \\
\text { necessidade de novos estudos } \\
\text { com um delineamento mais } \\
\text { precisos para lançar algumas } \\
\text { hipóteses de condução do } \\
\text { tratamento. Acrescido a isso, foi } \\
\text { visto a necessidade de } \\
\text { aumentar as capacitações e } \\
\text { formações dos cuidadores e dos } \\
\text { profissionais de saúde que irão } \\
\text { trabalhar de forma direta com os } \\
\text { idosos. }\end{array}$ \\
\hline
\end{tabular}

Fonte: Elaborado pelo autor (2021).

A doença de Alzheimer é uma afeção neurodegenerativa progressiva e irreversível, que inicialmente provoca a perda da memória recente e depois afeta os sentidos motores, cognitivos e intelectual (BATISTA, 2018). O Alzheimer pode ser diagnosticado por estágios, o que ajuda ao cirurgião-dentista no desenvolvimento do plano de tratamento. Nos estágios iniciais, o paciente desenvolve alguns sintomas como, problemas na cognição, dificuldades de memória leve, sinais de depressão ou ausência de estímulo e presença de irritabilidade com frequência (OLIVEIRA; D’ÁVILA, 2019). Nos estágios intermediários, esses sinais se agravam e são associados a outras manifestações, como, alucinações. Já nos estágios severos, os pacientes sofrem com esses problemas associados a doenças sistêmicas (ABRITTA; MIRANDA, 2012).

Além disso, observamos que a saúde geral está ligada diretamente da bucal, ou seja, as duas devem estar em sincronia para o bem-estar do indivíduo. Segundo Warmling et al (2016), os pacientes que possuem DA, geralmente, estão mais susceptíveis a desenvolver doenças periodontais ou lesões bucais, pela ausência dos cuidados básicos de higiene. As lesões mais presentes nos idosos são, candidíase, língua saburrosa, lesões pela prótese mal adaptada, úlceras, hiperplasia 
inflamatória, cáries dentárias extensas e raízes residuais (SPEZZIA, 2015). A maioria dessas manifestações estão ligadas ao Alzheimer pela carência dos cuidados com a higiene bucal, pelas manifestações sistêmicas associadas com as doenças bucais, pela falta do diagnóstico correto e de profissionais preparados para o atendimento odontológico.

Logo, para evitar ou minimizar algumas manifestações, é necessário realizar o controle das bactérias anaeróbias gram-positivas e gram-negativas, por meio de intervenções educativas de higiene bucal, ações preventivas, conscientização dos cuidados/familiares sobre a importância da saúde bucal e se preciso, usar dispositivos que auxiliem na desorganização do biofilme, ou seja, utilização de escovas elétricas, uso de colutórios sem álcool, visitas constantes ao dentista para realização de procedimentos básicos e educativos como, profilaxias, raspagens, restaurações primárias e acompanhamento da saúde bucal do idoso (ABRITTA GURGEL; MIRANDA, 2012).

Segundo Oliveira; D’ávila (2019), uma das maiores dificuldades que o cirurgião-dentista enfrenta durante os atendimentos aos pacientes com DA é devido a parte emocional do indivíduo, ou seja, por conta da destruição gradativa do hipocampo, o que impossibilita o paciente notificar as alterações bucais e acaba prejudicando as chances de cooperação do paciente. Além disso, o que acaba predicando é a dificuldade de permanência do paciente até a conclusão do tratamento, as doenças sistêmicas aliadas as manifestações bucais, a condição financeira e até mesmo, o incentivo familiar durante os tratamentos (VARJÃO, 2006).

Devido a essas dificuldades, alguns manejos podem ser desenvolvidos durante os atendimentos com estratégias preventivas rápidas e terapêuticas como, diagnóstico correto das fases da DA, criação de um ambiente confortável e confiável diante do idoso, uso de dispositivos auxiliares como, abridores de boca, fones de ouvido, espelhos externos, televisões, atendimentos dinâmicos com musicoterapia para deixar o ambiente mais familiar e procedimentos rápidos, humanizados e com respostas precisas, o que irá facilitar o desenvolvimento do atendimento (SANTIAGO et al., 2008).

Com isso, vale ressaltar, a importância da prevenção das doenças bucais, pois altera de forma direta a saúde do paciente, desenvolvendo doenças de modo 
sistêmico associado as doenças bucais (VARJÃO, 2006). Portanto, o cirurgiãodentista deve intervir de forma paliativa e curativa, a fim de colaborar com a qualidade e a expectativa de vida do idoso (SOUZA et al., 2006). Aliado a isso, para um tratamento ter êxito é necessário o uso de uma equipe multiprofissional e com procedimentos preventivos e minimamente invasivos com propostas curativas e preventivas para zelar pela vida dos pacientes com DA (OLIVEIRA; D'ÁVILA, 2019).

É evidente a importância de o cirurgião-dentista ter um conhecimento voltado para a DA, sendo necessário a aplicação de técnicas e manejos adequados para a condução do tratamento odontológico, tornando o atendimento satisfatório e garantindo a saúde bucal e a qualidade de vida do paciente.

Acrescido a isso, sugere-se mais estudos nessa linha de pesquisa para expandir esse horizonte, que compreenda o desenvolvimento do Alzheimer e os impactos que geram na vida do paciente, visto que eles são afetados de várias formas, como também desenvolver protocolos com uma perspectiva mais robusta e específica ao atendimento de pacientes com DA para que possa ser realizados diagnósticos precoces, o que irá auxiliar em um melhor prognóstico dos pacientes.

\section{CONSIDERAÇÕES FINAIS}

Está revisão integrativa possibilitou identificar que embora haja estudos e protocolos voltados ao tratamento odontológico e a doença de Alzheimer (DA), se faz necessário um protocolo mais robusto e objetivo que oriente o profissional de odontologia para o manejo adequado do paciente com a DA, permitindo assim uma maior uniformidade e qualidade nesse atendimento.

Em suma, os cirurgiões-dentistas devem estar preparados para atender os pacientes com Alzheimer, oferecendo um tratamento adequado e humanizado, para garantir um acompanhamento de qualidade e seguro aos pacientes com a DA, como também se faz necessário orientar os cuidadores e familiares, para a manutenção da saúde bucal desses idosos, proporcionando assim, uma melhor qualidade de vida. 
Dessa forma, é valido que o profissional aplique as técnicas e manejos mais adequados, com o auxílio dos dispositivos auxiliares, para tornar o atendimento odontológico no consultório confortável e individualizado para cada idoso, buscando sempre novos estudos e técnicas, além das qualificações continuadas nessa área.

\section{REFERÊNCIAS BIBLIOGRÁFICAS}

ABRITTA, G.; ANA, L.; MIRANDA, A. F. Atendimento multidisciplinar em paciente idosa com Alzheimer em fase intermediária em domicílio: Relato de caso: o cirurgião-dentista como integrante. Revista portal de divulgação, [s. I.], n. 20, 2012.

BARBOSA, É. P.; MENEZES, P. V. S.; NOVAES, C. R. M. N; LEANDRO, A. R. L; ASSIS, V. L. B.; REIS, M. C. S. Práticas de saúde oral em idosos com demência: Revisão sistemática. Research, Society and Development, [s. I.], v. 10, n. 9, 2021.

BATISTA, J. O. Uso de Novas Tecnologias no Controle da Doença de Alzheimer. Revista Científica Multidisciplinar Núcleo do Conhecimento. E. 06, v. 07, 2018.

BURLÁ, C. et al. Panorama prospectivo das demências no Brasil: um enfoque demográfico. Ciência \& saúde coletiva, [s. I.], 2013.

DIAS, M. H. M. S.; FONSECA, S. C. O atendimento de pacientes com a doença de Alzheimer na clínica odontológica: desafios e diretrizes. Revista Sociedade Brasileira de Geriatria e Gerontologia. São Paulo, 2011.

FRIEDLANDER, A. H. et al. Doença de Alzheimer: psicopatologia, tratamento médico e implicações odontológicas. The Journal of the American Dental Association, [s. I.], v. 137, ed. 9, p. 1240-1251, 2006. Disponível em: https://doi.org/10.14219/jada.archive.2006.0381. Acesso em: 10 nov. 2021.

GALLISA, M. C. Alzheimer na clínica odontológica. Revisão de literatura. Brasília - DF, 2006.

MIRANDA, A. F. et al. Doença de Alzheimer: características e orientações em odontologia. Revista Gaúcha de Odontologia. Porto Alegre, v. 58, 2010.

OLIVEIRA, M. C; D'ÁVILA, O. P. Guia de atenção à saúde bucal da pessoa com deficiência. Cartilha do Ministério da Saúde, Brasília. E. MS, 2019. 122p.

SANTIAGO E. et al. A saúde oral na doença de Alzheimer. Faculdade de Medicina Dentária da Universidade do Porto, 2008.

SERENIKI, A.; VITAL, M. A. B. F. A doença de Alzheimer: Aspectos fisiopatológicos e farmacológicos. Revista de Psiquitria do Rio Grande do Sul, Porto Alegre, p. 1-17, 18 dez. 2008. DOI https://doi.org/10.1590/S0101-81082008000200002. Disponível em: https://www.scielo.br/j/rprs/a/LNQzKPVKxLSsjbTnBCps4XM/abstract/?lang=pt. Acesso em: 10 nov. 2021.

SOUZA, A. C. A. R.; ROSA, C. F. B. D.; ELIAS, R. Doença de Alzheimer: protocolo de atendimento odontológico. Cispre. p. 1, 2006. 
SPEZZIA, S. Demência e saúde bucal. Revista da Faculdade de Ciências Médicas de Sorocaba, v. 17, n. 4, p. 175-178, 2015.

VARJÃO, F. M. Assistência odontológica para o paciente portador da doença de alzheimer. Revisão de literatura, Revista odonto ciência/PUCRS, v. 21, n. 53, 2006. p. 284-288, 3 out. 2006.

WARMLING, A. M. F. et al. Estratégias de cuidado bucal para idosos com doença de Alzheimer no domicílio. Revista Brasileira de geriatria e gerontologia, Rio de Janeiro, v. 19, ed. 5, 2016. 